\title{
On the Nonlinear Effects of Magnetoacoustic Perturbations in Optically Thin Quasi-Isentropic Plasmas
}

\author{
A. Perelomova* and F. Ratkowski \\ Gdańsk University of Technology, Faculty of Applied Physics and Mathematics, \\ G. Narutowicza 11/12, 80-233 Gdańsk, Poland \\ (Received April 4, 2017; in final form November 2, 2017)
}

\begin{abstract}
Nonlinear effects of planar magnetosound perturbations in a plasma are discussed. Plasma is non-adiabatic due to optically thin radiation and external heating. For these reasons, thermal instability of a plasma may appear which makes it acoustically active. The plasma is assumed to be initially homogeneous ideal gas with infinite electrical conductivity permeated by a straight magnetic field which is orthogonal to the trajectories of gas particles. The instantaneous dynamic equations which describe nonlinear effects of intense sound in quasi-isentropic plasma, are derived. Nonlinear interaction of periodic and aperiodic magnetoacoustic perturbations with the non-wave modes, are discussed. The conclusions concern dissipative or active behavior of magnetoacoustic perturbations which is determined by the kind of the heating-cooling function.
\end{abstract}

DOI: 10.12693/APhysPolA.133.194

PACS/topics: $43.25 .-\mathrm{x}, 43.25 . \mathrm{Nm}$

\section{Introduction}

The study of stable and unstable magnetohydrodynamic (MHD) perturbations of different spacial scales in a plasma are of the great importance. Thermal instability is observed in dynamics of astrophysical plasmas of different scales, including interstellar clouds and solar prominence formation. It is important in fluid flows in tokamaks. Non-adiabaticity along with the external heating of a plasma makes it acoustically active under some conditions [1]. That means that magnetoacoustic perturbations intensify in the course of propagation. This phenomenon relates to some kind of anomalous thermodynamical relaxation and following it dispersion. Relaxation of thermodynamic processes often leads to the similar properties of sound in fluids. We may mention gases with excited vibrational degrees of molecules and chemically reacting gases which thermal balance depends on some heat-loss function. This function includes in general external heating and cooling due to radiation and other reasons. In a plasma, this function may include a part responsible for the radiation cooling. An external source of energy causes deviation of the gas flow from isentropic, along with mechanical and thermal attenuation. Namely the isentropic instability is a reason for the acoustic waves to amplify in the course of propagation [2]. The irreversible processes and following them acoustical activity of a medium are of major interest in many applications concerning the solar chromosphere, interstellar gases and planetary nebulae. The nonlinear dynamics of magnetoacoustic waves in a thermally active and dissipative plasma has been studied analytically

*corresponding author; e-mail: anpe@mif.pg.gda.pl by Chin and co-authors in Ref. [3]. Authors of Ref. [3] have derived the generic nonlinear evolutionary equation which describes perturbations in active medium and have concluded about possibility of self-organization of MHD disturbances. In particular, it has been discovered that the formation of shock waves depends strongly on the type of the heating-cooling function. Discontinuities in the waveforms in a plasma may be supressed or form faster. The slow magnetoacoustic shock autowaves were described theoretically. Their magnitude is completely prescribed by the thermodynamic properties of equilibrium plasma and hence are independent on the initial or boundary conditions [4]. The effects of thermal radiation on the shock wave were considered in details also in Refs $[5,6]$. Flows over other relaxing media reveal the analogous features which are described by the similar equations [7].

The nonlinear interaction of magnetohydrodynamic waves of different kinds has been considered by numerous authors [8-10]. As for the nonlinear interaction of MHD waves with the non-wave modes, the subject is much less investigated. One may expect that the magnetoacoustic heating and streaming reveal anomalous behavior in the acoustically active plasma. That happens to all acoustically active media independently on the physical reason of acoustical activity [7, 11]. This study considers magnetoacoustic heating in a plasma with the generic heating-cooling function. This is enhancement of the entropy mode which follows propagation of sound in a nonlinear flow with attenuation of any kind $[12,13]$. As far as the authors know, magnetoacoustic heating is still unresolved issue in magnetic hydrodynamics. With regard to external heating or cooling, the nonlinear coupling of sound and the entropy mode is possible due to dispersion and correspondent attenuation (or amplification) of sound. The reason for that is dependence of the heating-cooling function on the thermodynamic quanti- 
ties. We do not consider mechanical and thermal losses of a plasma and its finite electrical conductivity. They introduce additional attenuation and dispersion in a fluid flow. Magnetoacoustic streaming and heating which are associated with these mechanisms, have been studied by one of the authors in Refs $[14,15]$. In this study, we derive the instantaneous dynamic equations responsible for excitation of the entropy and magnetic modes by sound and discuss them.

As for the nonlinear and dispersive distortions of the MHD perturbations theirselves, they have been wellunderstood analytically. Account for the second order derivatives, and, as consequence, for the quadratic nonlinearity in the heating-cooling function, "introduces new physics such as the existence of solitary waves" [1]. As usual, we consider a weak nonlinearity in this study. That means that the effects of nonlinearity may be obtained as corrections to the linear results. This concerns the dynamic equations. Nevertheless, these corrections are crucial in the description of nonlinear interactions. Nonlinear correction in the dynamic equation for wave perturbations may be also considered as a result of self-interaction of sound. Even in the case of small nonlinearity, it accumulates in time and is responsible for formation of shock fronts, enrichment of perturbations' spectrum and other manifestations of non-linear behavior. The effects of boundaries of a plasma are not considered. All evaluations are valid with accuracy up to quadratic nonlinear terms, that is, up to terms proportional to the squared Mach number, $M^{2}$.

\section{MHD modes of a planar flow}

The system of PDE equations consists of continuity equation, momentum equation, electrodynamic equations and energy balance equation

$$
\begin{aligned}
& \frac{\partial \rho}{\partial t}+\nabla \cdot(\rho \boldsymbol{v})=0, \\
& \rho \frac{\mathrm{d} \boldsymbol{v}}{\mathrm{d} t}=-\nabla p+\mu_{0}(\boldsymbol{\nabla} \times \boldsymbol{H}) \times \boldsymbol{H}, \\
& \frac{\partial \boldsymbol{H}}{\partial t}=\boldsymbol{\nabla} \times(\boldsymbol{v} \times \boldsymbol{H}), \quad \boldsymbol{\nabla} \cdot \boldsymbol{H}=0, \\
& \frac{\mathrm{d} p}{\mathrm{~d} t}-\gamma \frac{p}{\rho} \frac{\mathrm{d} \rho}{\mathrm{d} t}=(\gamma-1) L(p, \rho),
\end{aligned}
$$

where $\boldsymbol{v}, \boldsymbol{H}, p$, and $\rho$ are the plasma velocity, the magnetic field strength, pressure, density, respectively, and $\mu_{0}$ is the magnetic permeability of free space. $L(p, \rho)$ is the heating-cooling function which disturbs adiabaticity of fast perturbations in a plasma. Following Ref. [1], it is assumed to be dependent on pressure and density. The last equation in the set (2.1) relates to an ideal gas with the caloric equation of state

$$
e=C_{v} T=\frac{p}{(\gamma-1) \rho},
$$

where $e$ denotes the internal energy of a gas, and $\gamma=$
$C_{p} / C_{v}$ is the ratio of specific heats under constant pressure and constant density.

We are interested in weakly nonlinear equations, assuming the magnetic field $\boldsymbol{H}=(0,0, H(x, t))$ orthogonal to the velocity of gas particles $\boldsymbol{v}=(v(x, t), 0,0)$. The starting point represents the leading-order equations which follow from Eqs. (2.1):

$$
\begin{aligned}
& \frac{\partial \rho^{\prime}}{\partial t}+\rho_{0} \frac{\partial v}{\partial x}=-\rho^{\prime} \frac{\partial v}{\partial x}-v \frac{\partial \rho^{\prime}}{\partial x}, \\
& \frac{\partial v}{\partial t}+\frac{1}{\rho_{0}} \frac{\partial p^{\prime}}{\partial x}+\frac{1}{\rho_{0}} \frac{\partial h^{\prime}}{\partial x}=-v \frac{\partial v}{\partial x}+\frac{\rho^{\prime}}{\rho_{0}^{2}} \frac{\partial p^{\prime}}{\partial x}+\frac{\rho^{\prime}}{\rho_{0}^{2}} \frac{\partial h^{\prime}}{\partial x}, \\
& \frac{\partial p^{\prime}}{\partial t}+c^{2} \rho_{0} \frac{\partial v}{\partial x}-(\gamma-1)\left(L_{p} p^{\prime}+L_{\rho} \rho^{\prime}\right)= \\
& \quad-v \frac{\partial p^{\prime}}{\partial x}-\gamma p^{\prime} \frac{\partial v}{\partial x}+(\gamma-1)\left(0.5 L_{p p} p^{\prime 2}\right. \\
& \left.\quad+0.5 L_{\rho \rho} \rho^{\prime 2}+L_{p \rho} p^{\prime} \rho^{\prime}\right), \\
& \frac{\partial h^{\prime}}{\partial t}+2 h_{0} \frac{\partial v}{\partial x}=-v \frac{\partial h^{\prime}}{\partial x}-2 h^{\prime} \frac{\partial v}{\partial x},
\end{aligned}
$$

where $h$ denotes the magnetic pressure,

$$
h=\mu_{0} H^{2} / 2 \text {, }
$$

and

$$
\begin{aligned}
& L_{p}=\frac{\partial L}{\partial p}, \quad L_{\rho}=\frac{\partial L}{\partial \rho}, \quad L_{p p}=\frac{\partial^{2} L}{\partial p^{2}}, \\
& L_{\rho \rho}=\frac{\partial^{2} L}{\partial \rho^{2}}, \quad L_{p \rho}=\frac{\partial^{2} L}{\partial p \partial \rho}
\end{aligned}
$$

are partial derivatives of the heating-cooling function $L(p, \rho)$ evaluated at equilibrium state $\left(p_{0}, \rho_{0}\right)$. All variables represent a sum of unperturbed quantity, marked by subscript $0\left(v_{0}=0\right)$, and a disturbance, which is primed.

\subsection{Modes in a linear flow. Case $L_{p} \neq 0$}

As usual, establishment of the dispersion relations is the primary procedure in linear flows of a fluid. The dispersion relations follow immediately from the linearized Eqs. (2.2). All perturbations are thought as a sum of planar waves proportional to $\exp (\mathrm{i} \omega(k) t-i k x)$, where $k$ designates the wave number of any individual planar wave:

$$
f^{\prime}(x, t)=\int_{-\infty}^{\infty} \tilde{f}(k) \exp (\mathrm{i} \omega(k) t-i k x) \mathrm{d} k,
$$

$(\widetilde{f}(k) \exp (\mathrm{i} \omega(k) t)=\widetilde{f}(k, t)$ denotes the Fourier transform of $f^{\prime}(x, t)$, so as $\left.\widetilde{f}(k, t)=\frac{1}{2 \pi} \int_{-\infty}^{\infty} f(x, t) e^{\mathrm{i} k x} \mathrm{~d} x\right)$. The dispersion relations in a planar flow of a magnetic fluid reflect the solvability of Eq. (2.2). They take the forms

$$
\omega_{1,2}= \pm c_{m} k-\frac{i(\gamma-1)}{2 c_{m}^{2}}\left(c^{2} L_{p}+L_{\rho}\right), \quad \omega_{3}=0,
$$




$$
\omega_{4}=\frac{\mathrm{i}(\gamma-1)}{c_{m}^{2}}\left(L_{\rho}-\left(c_{m}^{2}-c^{2}\right) L_{p}\right),
$$

where

$$
c_{m}=\sqrt{c^{2}+c_{A}^{2}}, \quad c=\sqrt{\gamma \frac{p}{\rho}}, \quad c_{A}=\sqrt{2 h / \rho}
$$

designate the magnetosonic speed, the sound speed in an unmagnitized gas, and the Alfvén speed, respectively, evaluated at the equilibrium state $\left(p_{0}, \rho_{0}\right)$.

The first two roots $\omega_{1}, \omega_{2}$ relate to the magnetosonic waves of different directions of propagation (so-called fast MHD waves), the third root $\omega_{3}$ corresponds to the magnetic Alfvén mode in the flow where magnetic field is perpendicular to the particles velocity, and the last one, $\omega_{4}$, corresponds to the entropy mode. The dispersion relations in Eqs. (2.3) are calculated with accuracy up to terms proportional to the first powers of $L_{p}, L_{\rho}$. We arrive at the conclusion that the medium is acoustically active under the condition

$$
c^{2} L_{p}+L_{\rho}>0 \text {, }
$$

which has been discovered in the studies of Nakariakov and co-authors. In this case, MHD perturbations enhance in the course of propagation. The total perturbation is represented by a sum of specific disturbances which in fact form eigenvectors of correspondent matrix operator:

$$
\begin{aligned}
v & =\sum_{i=1}^{4} v_{i}=\frac{c_{m}}{\rho_{0}} \rho_{1}-\frac{(\gamma-1)\left(c^{2} L_{p}+L_{\rho}\right)}{2 c_{m}^{2} \rho_{0}} \int \rho_{1} \mathrm{~d} x \\
& -\frac{c_{m}}{\rho_{0}} \rho_{2}-\frac{(\gamma-1)\left(c^{2} L_{p}+L_{\rho}\right)}{2 c_{m}^{2} \rho_{0}} \int \rho_{2} \mathrm{~d} x+ \\
& \frac{(\gamma-1)\left(L_{\rho}-\left(c_{m}^{2}-c^{2}\right) L_{p}\right)}{c_{m}^{2} \rho_{0}} \int \rho_{4} \mathrm{~d} x, \\
p^{\prime} & =\sum_{i=1}^{4} p_{i}=c^{2} \rho_{1}-\frac{(\gamma-1)\left(c^{2} L_{p}+L_{\rho}\right)}{c_{m}} \int \rho_{1} \mathrm{~d} x \\
& +c^{2} \rho_{2}+\frac{(\gamma-1)\left(c^{2} L_{p}+L_{\rho}\right)}{c_{m}} \int \rho_{2} \mathrm{~d} x-\frac{L_{\rho}}{L_{p}} \rho_{3} \\
& +\left(c^{2}-c_{m}^{2}\right) \rho_{4}, \\
h^{\prime} & =\sum_{i=1}^{4} h_{i}=\left(c_{m}^{2}-c^{2}\right) \rho_{1}+\left(c_{m}^{2}-c^{2}\right) \rho_{2}+\frac{L_{\rho}}{L_{p}} \rho_{3} \\
& +\left(c_{m}^{2}-c^{2}\right) \rho_{4} .
\end{aligned}
$$

Index in summation $i$ denotes the ordering number of specific mode. The entropy mode $(i=4)$ is isobaric in the sense that summary pressure, which consists of thermodynamic and magnetic parts, keeps constant. This is valid also for the Alfvén mode with ordering number $i=3$. In contrast to the newtonian flow, the entropy mode possesses variations in pressure so that the correspondent variations in temperature of the background associated with this mode, should be evaluated accurately.
The rows which distinguish excess densities corresponding to third and fourth roots,

$$
P_{3}\left(\begin{array}{c}
\rho^{\prime} \\
v \\
p^{\prime} \\
h^{\prime}
\end{array}\right)=\rho_{3}, \quad P_{4}\left(\begin{array}{c}
\rho^{\prime} \\
v \\
p^{\prime} \\
h^{\prime}
\end{array}\right)=\rho_{4},
$$

take the forms:

$$
\begin{gathered}
P_{3}=\left(\begin{array}{c}
1+\frac{L_{\rho}}{\left(c_{m}^{2}-c^{2}\right) L_{p}} \\
0 \\
0 \\
-\frac{1}{c_{m}^{2}-c^{2}}-\frac{L_{\rho}}{\left(c_{m}^{2}-c^{2}\right)^{2} L_{p}}
\end{array}\right)^{\mathrm{T}}, \\
P_{4}=\left(\begin{array}{c}
-\frac{L_{\rho}}{\left(c_{m}^{2}-c^{2}\right) L_{p}} \\
-\frac{\left.(\gamma-1) \rho_{0} c^{2} L_{p}+L_{\rho}\right)}{c_{m}^{4}} \int \mathrm{d} x \\
-\frac{1}{c_{m}^{2}} \\
\frac{c^{2}}{c_{m}^{2}\left(c_{m}^{2}-c^{2}\right)}+\frac{L_{\rho}}{\left(c_{m}^{2}-c^{2}\right)^{2} L_{p}}
\end{array}\right)^{\mathrm{T}} .
\end{gathered}
$$

They are evaluated with accuracy up to terms proportional to the first powers of $L_{p}$ and $L_{\rho}$. The limits of integration depend on the physical context of a flow. When $P_{3}, P_{4}$ apply at the linearized Eqs. (2.2), they reduce all therms containing the foreign perturbations and yield the linear dynamic equations which govern $\rho_{3}$ and $\rho_{4}$, respectively. These equations contain the first order partial derivatives with respect to time. In the following section, we consider the non-linear interaction of magnetoacoustic waves with the non-wave modes in the planar flow.

\section{Nonlinear effects which associate with the intense magnetoacoustic wave}

Application of $P_{3}$ and $P_{4}$ at the system (2.2), which includes quadratic nonlinear terms, leads to weakly nonlinear evolutionary equations with properly distributed quadratic nonlinear terms. Usually, among all variety of nonlinear terms, these pure magnetoacoustic are of major importance. That corresponds to the intense perturbations in MHD waves as compared with those of the non-wave modes at some temporal and spacial domains.

For definiteness, the first MHD mode, which propagates in the positive direction of axis $O X$, will be considered. It relates to $\omega_{1}$ from Eqs. (2.3). For correct description of effects associating with quadratic nonlinear terms, the linear relations should be corrected by means of involving terms which make MHD wave isentropic in the leading order [15]. The corrected links are as follows

$$
\psi_{1, n}=\left(\begin{array}{l}
\rho_{1} \\
v_{1} \\
p_{1} \\
h_{1}
\end{array}\right)+\left(\begin{array}{c}
\frac{c_{m}^{2}-c^{2}(\gamma-2)}{4 c_{m}^{4}} \rho_{0} \\
0 \\
\frac{c^{2}\left(c_{m}^{2}(2 \gamma-1)-c^{2}(\gamma-2)\right)}{4 c_{m}^{4}} \rho_{0} \\
\frac{\left(c_{m}^{2}-c^{2}\right)\left(3 c_{m}^{2}-c^{2}(\gamma-2)\right)}{4 c_{m}^{2}} \rho_{0}
\end{array}\right) v_{1}^{2} .
$$

In the absence of magnetic field and inflow of external energy, these corrections equal the well-known terms which make the progressive the Riemann wave isentropic [12]. 
The equation governing velocity in the first magnetoacoustic planar wave which propagates in the positive direction of axis $O x$, takes the form:

$$
\begin{gathered}
\frac{\partial v_{1}}{\partial t}+c_{m} \frac{\partial v_{1}}{\partial x}-\frac{(\gamma-1)}{2 c_{m}^{2}}\left(c^{2} L_{p}+L_{\rho}\right) v_{1} \\
+\frac{3 c_{m}^{2}+c^{2}(\gamma-2)}{2 c_{m}^{2}} v_{1} \frac{\partial v_{1}}{\partial x}=0 .
\end{gathered}
$$

Eq. (3.1) describes nonlinear propagation of the MHD wave in a magnetic gas. It is similar to dynamic equations which describe wave perturbations in other media which may be acoustically active [7]. Eq. (3.1) without account for heating-cooling function has been firstly derived and analyzed in the context of propagation of a saw-tooth impulse in Ref. [16]. Eq. (3.1) may be readily transformed into the leading-order pure nonlinear equation

$$
\frac{\partial V_{1}}{\partial X}-\frac{3 c_{m}^{2}+c^{2}(\gamma-2)}{2 c_{m}^{4}} V_{1} \frac{\partial V_{1}}{\partial \tau}=0,
$$

by means of new variables

$$
\begin{aligned}
V_{1} & =v_{1} \exp \left(-\frac{(\gamma-1)}{2 c_{m}^{3}}\left(c^{2} L_{p}+L_{\rho}\right) x\right), \\
X & =-\frac{2 c_{m}^{3}}{(\gamma-1)\left(c^{2} L_{p}+L_{\rho}\right)} \\
& \times\left(1-\exp \left(\frac{(\gamma-1)}{2 c_{m}^{3}}\left(c^{2} L_{p}+L_{\rho}\right) x\right)\right), \\
\tau & =t-x / c
\end{aligned}
$$

Eq. (3.2) may be solved by the method of characteristics.

The main conclusion is that the shock wave always forms in acoustically active media and may not arise otherwise due to attenuation [7]. Eq. (3.1) coincides with that derived in Ref. [3]. Eq.(15) therein describes propagation of magnetoacoustic perturbations for any direction of the straight magnetic field and includes effects by thermal conductivity and nonlinear term associated with the heating-cooling function. Eq. (3.1) represents the particular case of Eq. (15) from Ref. [3] with $\theta=\pi / 2$, zero thermal conduction and zero nonlinear term associated with the heating-cooling function. Chin and co-authors in Ref. [3] include heating and radiative cooling but consider perturbations over constant background. The validity of this approach should be carefully investigated in view of that the external source makes the background temperature nonuniform. Namely, that follows from the energy balance for the thermoconducting fluid in the zero order. The non-uniformity of the background may essentially affect the wave dynamics, especially at low frequencies. This is the case when the characteristic length of non-uniformity is of order or smaller than the wave length.

By means of projecting Eqs. (2.1) into the different subspaces which are designated by dispersion relations, one readily obtains equations which describe dynamics of $\rho_{3}$ or $\rho_{4}$. The rows $P_{3}$ and $P_{4}$ project the system onto rel- ative dynamic equations, eliminating all foreign terms in the linear part of the final equations. Among all variety of quadratic nonlinear terms, only MHD terms belonging to the first mode, will be kept. They form the "magnetoacoustic forces" of the secondary modes. As the result of application of $P_{3}$, one arrives at the equation which governs an excess density in the Alfvén mode:

$$
\begin{aligned}
& \frac{\partial \rho_{3}}{\partial t}=\frac{\rho_{0}(\gamma-1)}{2 c_{m}^{4}\left(c_{m}^{2}-c^{2}\right)}\left(c^{2}\left(c_{m}^{2}-c^{2}\right) L_{p}+c_{m}^{2} L_{\rho}\right) \\
& \quad \times\left(v_{1}^{2}+\frac{\partial v_{1}}{\partial x} \int v_{1} \mathrm{~d} x\right) .
\end{aligned}
$$

For periodic magnetoacoustic perturbations, in the leading order,

$$
\overline{v_{1}^{2}}=-\overline{\frac{\partial v_{1}}{\partial x} \int v_{1} \mathrm{~d} x}
$$

where top line denotes the temporal average over period of the acoustic wave, and Eq. (3.3) possesses approximately zero right-hand acoustic source. Acoustic heating is described by equation wich results when $P_{4}$ applies at the system Eqs. (2.2). We reproduce it averaged over the sound period form in the case of nearly periodic magnetoacoustic perturbations:

$$
\begin{aligned}
& \frac{\overline{\partial \rho_{4}}}{\partial t}+\frac{(\gamma-1)}{c_{m}^{2}}\left(L_{\rho}-\left(c_{m}^{2}-c^{2}\right) L_{p}\right) \overline{\rho_{4}}= \\
& \frac{\rho_{0}(\gamma-1)}{4 c_{m}^{6}}\left(c^{4}\left(3(\gamma-2) L_{p}-2 c_{m}^{2} \rho_{0} L_{p p}\right)+\right. \\
& c_{m}^{2}\left(L_{\rho}+4 \gamma L_{\rho}-2 \rho_{0} L_{\rho \rho}\right)
\end{aligned}
$$

$\left.+c^{2}\left(3(\gamma-2) L_{\rho}+c_{m}^{2}\left(3 L_{p}+2 \gamma L_{p}-4 \rho_{0} L_{p \rho}\right)\right)\right) \overline{v_{1}^{2}}$,

which rearranges in the case of approximately equal $c_{m}$ and $c$ into the following equation:

$$
\begin{aligned}
& \frac{\overline{\partial \rho_{4}}}{\partial t}+\frac{(\gamma-1)}{c_{m}^{2}}\left(L_{\rho}-\left(c_{m}^{2}-c^{2}\right) L_{p}\right) \overline{\rho_{4}} \equiv I_{1}= \\
& \frac{\rho_{0}(\gamma-1)}{4 c^{4}}\left(c^{2}(5 \gamma-3) L_{p}+(7 \gamma-5) L_{\rho}\right. \\
& \left.-2 c^{4} \rho_{0} L_{p p}-4 c^{2} \rho_{0} L_{p \rho}-2 \rho_{0} L_{\rho \rho}\right) \overline{v_{1}^{2}},
\end{aligned}
$$

which is readily integrated with the result

$$
\begin{gathered}
\overline{\rho_{4}}=\exp \left(-\frac{(\gamma-1)}{c_{m}^{2}}\left(L_{\rho}-\left(c_{m}^{2}-c^{2}\right) L_{p}\right) t\right) \\
\times \int_{0}^{t} \exp \left(\frac{(\gamma-1)}{c_{m}^{2}}\left(L_{\rho}-\left(c_{m}^{2}-c^{2}\right) L_{p}\right) t^{\prime}\right) I_{1}\left(x, t^{\prime}\right) \mathrm{d} t^{\prime} .
\end{gathered}
$$

This solution corresponds to zero initial $\rho_{4}$. As for the acoustic streaming, it appears in flows exceeding one dimension. This is the bulk motion of a fluid caused by nonlinear loss in momentum of sound. The acoustic source of acoustic streaming follows from the momentum equation and depends on the type of attenuation. The projecting of the momentum equation becomes simpler in the case of quasi-planar flow. Let the velocity of a planar flow includes two components: $\boldsymbol{v}=$ 
$\left(v_{x}(x, y, t), v_{y}(x, y, t), 0\right)$. It is perpendicular to magnetic field $\boldsymbol{H}=\left(0,0, H_{z}(x, y, t)\right)$. The acoustic beam propagates for definiteness in the positive direction of axis $x$ and weakly diverges in the transversal direction. The magnetoacoustic force of streaming is in fact quadratic acoustic source that makes the longitudinal velocity of the vortex flow to increase in time. The averaged over the sound period equation, takes the form:

$$
\frac{\partial \overline{v_{x, v o r t}}}{\partial t}=F_{m, s}=-\frac{(\gamma-1)}{\rho_{0}^{2} c^{4} c_{m}^{2}}\left(c^{2} L_{p}+L_{\rho}\right) \overline{p_{1}^{2}} .
$$

In acoustically active plasma, $F_{m, s}$ is negative and, consequently, the direction of streamlines turns about as it happens to other acoustically active gases.

\subsection{The heating-cooling function which depends exclusively on temperature, $L(T)$}

It is useful to consider the case when the heatingcooling function depends exclusively on temperature, $L(T)$. Making use of equalities

$$
\begin{aligned}
& L_{p}=\frac{L_{T}}{C_{V}(\gamma-1) \rho_{0}}, \quad L_{\rho}=-\frac{c^{2} L_{T}}{C_{V}(\gamma-1) \gamma \rho_{0}}, \\
& L_{p p}=\frac{L_{T T}}{C_{V}^{2}(\gamma-1)^{2} \rho_{0}^{2}}, \\
& L_{p \rho}=-\frac{C_{V}(\gamma-1) \gamma L_{T}+c^{2} L_{T T}}{C_{V}^{2}(\gamma-1)^{2} \gamma \rho_{0}^{2}} \\
& L_{\rho \rho}=\frac{c^{2}\left(2 C_{V}(\gamma-1) \gamma L_{T}+c^{2} L_{T T}\right)}{C_{V}^{2}(\gamma-1)^{2} \gamma^{2} \rho_{0}^{2}}
\end{aligned}
$$

where

$$
\frac{d L}{d T} \equiv L_{T}, \quad \frac{d^{2} L}{d T^{2}} \equiv L_{T T}
$$

one may readily rearrange Eq. (3.3) into the dynamic equation

$$
\frac{\partial \rho_{3}}{\partial t}=-\frac{c^{2}\left(-c_{m}^{2}(\gamma-1)+\gamma c^{2}\right) L_{T}}{2 \gamma c_{m}^{4}\left(c_{m}^{2}-c^{2}\right) C_{V}}\left(v_{1}^{2}+\frac{\partial v_{1}}{\partial x} \int v_{1} \mathrm{~d} x\right),
$$

which equals approximately zero on average for nearly periodic sound. Variation in temperature $T_{3}$ which specifies the Alfvén mode, equals zero:

$$
\begin{gathered}
T_{3}=\frac{p_{3}}{C_{V}(\gamma-1) \rho_{0}}-\frac{p_{0} \rho_{3}}{C_{V}(\gamma-1) \rho_{0}^{2}}= \\
\frac{1}{C_{V}(\gamma-1) \rho_{0}}\left(p_{3}-\frac{c^{2}}{\gamma} \rho_{3}\right)=0
\end{gathered}
$$

in accordance to relations of $\rho_{3}$ and $p_{3}$ established by Eqs. (2.4) and equalities Eqs. (3.6). If $L_{T}>0$, a gas is acoustically active. Sound enhances in a medium taking energy from the background. That may be readily concluded from the acoustic dispersion relations, Eqs. (2.3):

$$
\begin{aligned}
& \omega_{1,2}= \pm c_{m} k-\frac{i(\gamma-1)}{2 c_{m}^{2}}\left(c^{2} L_{p}+L_{\rho}\right)= \\
& \pm c_{m} k-\frac{i(\gamma-1) c^{2}}{2 C_{V} \gamma \rho_{0} c_{m}^{2}} L_{T} .
\end{aligned}
$$

The equation for the excitation of the entropy mode fol- lows from making use of $P_{4}$. We reproduce its averaged form for the periodic MHD perturbations:

$$
\begin{aligned}
& \frac{\overline{\partial \rho_{4}}}{\partial t}-\frac{c^{2}+\gamma\left(c_{m}^{2}-c^{2}\right)}{\gamma c_{m}^{4} C_{V} \rho_{0}} L_{T} \overline{\rho_{4}}=I_{2} \equiv \\
& \frac{c^{2}(\gamma-1)}{4 \gamma^{2} c_{m}^{6} C_{V}^{2}}\left(c_{m}^{2} C_{V} \gamma(5+2 \gamma) L_{T}+c^{2}\left(3 C_{V}(\gamma-2) \gamma L_{T}\right.\right. \\
& \left.\left.\quad-2 c_{m}^{2} L_{T T}\right)\right) \overline{v_{1}^{2}},
\end{aligned}
$$

In the case of small difference between $c$ and $c_{m}$ (that is, for weak magnetic strength),

$$
I_{2}=\frac{\gamma-1}{4 \gamma^{2} c^{2} C_{V}^{2}}\left(C_{V}(5 \gamma-1) \gamma L_{T}-2 c^{2} L_{T T}\right) \overline{v_{1}^{2}} .
$$

The second-order derivative is not of importance, if

$$
\left|L_{T T}\right| \ll \frac{(5 \gamma-1) \gamma C_{V}}{2 c^{2}}\left|L_{T}\right|=\frac{\gamma^{2}(5 \gamma-1)}{2(\gamma-1) T_{0}}\left|L_{T}\right| .
$$

All terms should be evaluated at unperturbed thermodynamic state. The function $\frac{\gamma^{2}(5 \gamma-1)}{2(\gamma-1)}$ achieves minimum, approximately 14.6 at $\gamma=1.46$. Eq. (3.7) is readily integrated with the result

$$
\begin{aligned}
\overline{\rho_{4}} & =\exp \left(\frac{c^{2}+\gamma\left(c_{m}^{2}-c^{2}\right) L_{T}}{\gamma c_{m}^{4} C_{V} \rho_{0}} t\right) \\
& \times \int_{0}^{t} \exp \left(-\frac{c^{2}+\gamma\left(c_{m}^{2}-c^{2}\right) L_{T}}{\gamma c_{m}^{4} C_{V} \rho_{0}} t^{\prime}\right) I_{2}\left(x, t^{\prime}\right) \mathrm{d} t^{\prime} .
\end{aligned}
$$

This solution corresponds to zero initial $\rho_{4}$. It establishes negative production of excess density and positive production of excess temperature associating with the entropy mode in unit time, if $L_{T}<0$. These excess quantities are related as

$$
T_{4}=-\frac{c^{2}+\gamma\left(c_{m}^{2}-c^{2}\right)}{\gamma(\gamma-1) C_{V} \rho_{0}} \rho_{4} .
$$

$L_{T}>0$ corresponds to acoustically active gases and production of positive excess density specifying the entropy mode. That reflects decrease in the background temperature. The excess quantities of the entropy mode may considerably exceed the excess quantities which associate with the third Alfvén mode due to the factor $c_{m}^{2}-c^{2}$ in the link between $p_{4}$ and $\rho_{4}$.

\section{The case $L_{p}=0$}

If only $L_{\rho}$ differs from zero, both non-wave modes should be re-determined, as well as correspondent projectors. The dispersion relations take the limiting form Eq. (2.3) for $L_{p}=0$, the links of perturbations in the fourth entropy mode also is a limiting case of these given by Eqs. (2.4), but the Alfvén mode is specified by the relations which are not the limiting case:

$$
v_{3}(x, t)=\rho_{3}(x, t)=0, \quad h_{3}(x, t)=-p_{3}(x, t) .
$$

The projecting rows which distinguish $p_{3}$ and $\rho_{4}$, take the forms: 


$$
P_{3, p}=\left(\begin{array}{c}
c_{m}^{2}-c^{2} \\
0 \\
0 \\
-1
\end{array}\right)^{\mathrm{T}}, \quad P_{4}=\left(\begin{array}{c}
1 \\
-\frac{(\gamma-1) \rho L_{\rho}}{c_{m}^{m}} \int \mathrm{d} x \\
-\frac{1}{c_{m}^{2}} \\
-\frac{1}{c_{m}^{2}}
\end{array}\right)^{\mathrm{T}} .
$$

Applying of projectors result in the following leadingorder dynamic equations:

$$
\begin{aligned}
& \frac{\partial p_{3}}{\partial t}=\frac{\left(c_{m}^{2}-c^{2}\right)(\gamma-1) \rho_{0} L_{\rho}\left(v_{1}^{2}+\partial v_{1} / \partial x \int v_{1} \mathrm{~d} x\right)}{2 c_{m}^{4}}, \\
& \frac{\partial \rho_{4}}{\partial t}+\frac{(\gamma-1) L_{\rho}}{c_{m}^{2}} \rho_{4}^{\prime}=\frac{(\gamma-1)(7 \gamma-5) \rho_{0} L_{\rho}}{4 c^{4}} v_{1}^{2} .
\end{aligned}
$$

These equations are instantaneous. They both are easily integrated, with the solution of the second one in the form

$$
\begin{aligned}
\rho_{4} & =\frac{(\gamma-1)(7 \gamma-5) \rho_{0} L_{\rho}}{4 c^{4}} \exp \left(-\frac{(\gamma-1) L_{\rho}}{c_{m}^{2}} t\right) \\
& \times \int_{0}^{t} \exp \left(\frac{(\gamma-1) L_{\rho}}{c_{m}^{2}} t^{\prime}\right) v_{1}\left(x, t^{\prime}\right)^{2} \mathrm{~d} t^{\prime}
\end{aligned}
$$

which corresponds zero initial $\rho_{4}$. In the normal pumping of energy, $L_{\rho}<0$ and excess temperature specifying the fourth mode, is positive and enlarges in time. The excess quantities of density and temperature are related in accordance to Eq. (3.8).

\section{Concluding remarks}

This study brings out some features of excitation of the non-wave modes in the field of intense sound in the magnetic fluid. The balance of energy is described by the heating-cooling function $L$ which in general depends on pressure and density of a gas. The linear features of flows in open systems are well-understood. That concerns acoustical activity under some conditions which depend on the first partial derivatives of $L$ over pressure $p$ and density $\rho$. The recent studies consider also influence of the second partial derivatives of $L$ in connection with dynamics of MHD waves. As far as the authors know, nonlinear interaction of intense sound with nonwave modes in a plasma with input inflow of energy were not studied.

We have considered particular cases of the heatingcooling function:

1) $L_{p}=\frac{\partial L(p, \rho)}{\partial p} \neq 0$

2) $L$ depends exclusively on temperature;

3) $L_{p}=\frac{\partial L(p, \rho)}{\partial p}=0$.

Conditions of acoustical activity are established in all cases. Weakly nonlinear governing equations of the nonwave modes are derived which take into account acoustic sources. They are valid for periodic and aperiodic sound independently on the spectrum of magnetoacoustic disturbances. Instantaneous Eqs. (3.4), (4.1) are the main results of the study. They specify dynamics of an excess density in the entropy mode in the field of sound. As for the vortex flow which is induced in the field of intense sound, it is written on in the averaged form and is valid for all three particular cases of the heating-cooling function. This is Eq. (3.5). If $L$ depends exclusively on temperature, $c^{2} L_{p}+L_{\rho}=c^{2} L_{T} /\left(\gamma \rho_{0} C_{V}\right)$, and all second-order derivatives of $L(p, \rho)$ are easily expressed in terms of $\frac{d L}{d T}$ and $\frac{d^{2} L}{d T^{2}}$. This particular case is considered in Sec.3.1. Both magnetoacoustic heating and streaming manifest the non-uniformity of the background parameters of a plasma, that is, thermal lenses and bulk flows which follow attenuation or amplification of sound. These inhomogeneities in turn have impact on the sound propagation. Therefore, they may be of especial interest in the plasma's applications. As for the magnetic Alfvén mode, its production is insignificant, at least in the case of periodic or almost periodic MHD perturbations. The case $L_{p}=0$ corresponds to the isochoric Alfvén mode, and the case $L(T)$ specifies isotermal Alfvén mode.

The magnetoacoustic forces of the secondary non-wave modes are proportional to the sound intensity and depend on the magnetic strength. In general, properties of nonlinear flow in a plasma are very similar to other flows of gases which may be acoustically active, though the physical reasons for acoustical activity are various. The reason for acoustical activity of a plasma is determined by the kind of heating-cooling function, that is, by external inflow of energy which disturbs the adiabaticity of quick perturbations. There are no restrictions concerning strength of the magnetic field in this study. Magnetoacoustic perturbations may be periodic or not and may include waves of all wave numbers. The authors apply the method of projecting of initial system of conservation equations into the system of coupling equations for interacting modes independently on their initial spectrum. The results of this study may be addressed to a hot atomic plasma with temperature greater than $10^{4} \mathrm{~K}$ and a cold molecular gas with temperature less than $10^{3} \mathrm{~K}$ and to different kinds of the function $L(p, \rho)$ which describes effects of non-adiabaticity. It may take form of constant heating per unit of mass (this is the case of $L_{p}=0$, if losses due to radiation are left out of account) or heating specific for coronal current dissipation (this is the case of $L_{\rho}=0$ ) in the high-temperature gas. In the cold molecular gas, the external heating occurs mainly due to grain photoelectrons and cosmic rays [17]. This is the case of $L_{p}=0$. Generally, the radiation function also contributes in $L$. That has impact on partial derivatives of $L$ with respect to its arguments. In particular, the optically thin hot plasma radiates proportionally to $\rho^{2-\eta} p^{\eta}[18]$. The slow variations of perturbations in the non-wave modes may be measured remotely, and the kind of heating-cooling function in a plasma may be established analytically. This may be of importance in many applications. The results of this study may be applied in predictions of slow perturbations in temperature and density of plasma which follow magnitoacoustic wave, and in the inverse problems which associate with specification of magnetoacoustic perturbations, including aperiodic and pulses. 


\section{References}

[1] V.M. Nakariakov, C.A. Mendoza-Briceńo, M.H. Ibáńez, Astrophys. J. 528, 767 (2000).

[2] G.B. Field, Astrophys. J. 142, 531 (1965).

[3] R. Chin, E. Verwichte, G. Rowlands, V.M. Nakariakov, Phys. Plasmas 17, 107 (2010).

[4] A. Kelly, V.M. Nakariakov, SOHO 13 Waves, Oscillations and Small-Scale Transients Events in the Solar Atmosphere: Joint View from SOHO and TRACE, Ed. H. Lacoste, ESA Special Publication, Vol. 547, 2004, p. 483.

[5] L.P. Singh, R. Singh, S.D. Ram, Astrophys. Space Sci. 342, 371 (2012)

[6] R. Shyam, V.D. Sharma, J. Sharma, AIAA J. 19, 1246 (1981).

[7] A.I. Osipov, A.V. Uvarov, Sov. Phys. Usp. 35, 903 (1992).

[8] R.Z. Sagdeev, A.A. Galeev, Nonlinear Plasma Theory, Benjamin, New York 1969.
[9] V.I. Petviashvili, O.A. Pokhotelov, Solitary Waves in Plasmas and in the Atmosphere, Gordon and Breach, Berlin 1992, p. 1.

[10] P.K. Shukla, L. Stenflo, in: Nonlinear MHD Waves and Turbulence, Lecture Notes in Solar Phys., Eds. T. Passot, P.-L. Sulem, Springer, Berlin 1999, p. 1.

[11] N.E. Molevich, Acoust. Phys. 47, 102 (2001).

[12] O.V. Rudenko, S.I. Soluyan, Theoretical Foundations of Nonlinear Acoustics, Plenum, New York 1977.

[13] M. Hamilton, D. Blackstock, Nonlinear Acoustics, Academic Press, New York 1998.

[14] A. Perelomova, Acta Phys. Pol. A 130, 727 (2016).

[15] A. Perelomova, Archiv. Acoust. 41, 691 (2016).

[16] V.D. Sharma, L.P. Singh, R. Ram, Phys. Fluids 30, 1572 (1987)

[17] M.H. Ibáńez, S.A. Parravano, Astrophys. J. 424, 763 (1994)

[18] J.F. Vesecky, S.K. Antiochos, J.H. Underwood, Astrophys. J. 233, 987 (1979). 